\title{
STEP-BY-STEP INTEGRATION OF ORDINARY DIFFERENTIAL EQUATIONS*
}

\author{
$\mathrm{BY}$ \\ S. C. R. DENNIS** \\ Weizmann Institute of Science, Rehovot, Israel
}

1. Introduction. Finite-difference methods of integration of differential equations are usually based on the assumption that, locally, the wanted function may be represented by a polynomial function of the independent variable (or variables, in the case of partial differential equations). Polynomial assumption is not necessarily, however, the only starting point. Recently, Allen and Southwell (1) found it desirable to employ other types of approximating functions when dealing with certain second-order partial differential equations. Investigation of their approximations (2) suggests that they have substantial merit in certain cases. In the present paper we shall investigate a step-bystep integration process for ordinary differential equations which is based on Allen and Southwell's type of approximating function. An investigation of the type we consider makes it necessarily limited in detail. In fact, we shall limit detailed work to the important linear, second-order, initial value problem

with

$$
y^{\prime \prime}+p(x) y^{\prime}+q(x) y=r(x)
$$

$$
y\left(x_{0}\right)=y_{0}, \quad y^{\prime}\left(x_{0}\right)=y_{0}^{\prime},
$$

where primes denote differentiation with respect to the real variable $x$ and $p, q$ and $r$ are given functions of $x$. This problem occurs frequently enough to merit attention; and it should become clear that the process may be extended to many other important problems.

A convenient and simple special case of (1) with which to start is the equation

$$
y^{\prime \prime}+q(x) y=0
$$

where, in physical applications, $q(x)$ is often of the form $q(x)=\lambda \rho(x)-\sigma(x), \lambda$ being an eigenvalue. Step-by-step integration is a well-known process of constructing, by successive stages, a numerical approximation to the solution $y(x)$ over some range $x=x_{0}$ to $x=\zeta$. The range $\left(x_{0}, \zeta\right)$ is divided into a finite number of intervals, and the purpose of step-by-step formulae is to relate $y$ (and possibly also its derivative) at a given pivotal point with corresponding values at previous pivotal points. This defines a numerical solution, starting from $x=x_{0}$. For simplicity, let us suppose $q(x)$ continuous in $\left(x_{0}, \zeta\right)$ and to tend to finite limits at the end points. It is then known, (cf. Ince (3), de la Vallée Poussin (4)), that a unique solution of Equation (3) exists satisfying the end conditions (2). As usual suppose $\left(x_{0}, \zeta\right)$ divided into $n$ partial intervals $\left(x_{m-1}, x_{m}\right)$, $(m=1,2, \cdots, n)$, where $x_{n}=\zeta$. For simplicity, take these equal, i.e. $x_{m+1}-x_{m}=h$, although this is not necessary. If $q_{m}$ denote some value of $q(x)$ in $\left(x_{m-1}, x_{m}\right)$ consider the function $\phi_{n}(x)$ defined by means of the equation

$$
\phi_{n}^{\prime \prime}(x)+\psi_{n}(x) \phi_{n}(x)=0, \quad(0 \leq x \leq \zeta)
$$

*Received April 14, 1961.

**Now at the University of Sheffield. 
where

$$
\psi_{n}(x)=q_{m}, \quad\left(x_{m-1} \leq x \leq x_{m}\right), \quad(m=1,2, \cdots, n) .
$$

In the interval $\left(x_{m-1}, x_{m}\right)$ this function satisfies

$$
\phi_{n}^{\prime \prime}(x)+q_{m} \phi_{n}(x)=0,
$$

and an expression for $\phi_{n}(x)$, arbitrary to the extent of two constants of integration, can be written down. To complete the definition of $\phi_{n}(x)$ in $\left(x_{0}, \zeta\right)$ we choose these constants, $2 n$ in all, to make $\phi_{n}(x)$ and $\phi_{n}^{\prime}(x)$ continuous at each internal point of subdivision $x_{1}, x_{2}, \cdots, x_{n-1}$ and in addition to satisfy the initial conditions (2). The function $\phi_{n}(x)$ defined in this way is, with its derivative, continuous for all $x$ in $\left(x_{0}, \zeta\right)$; it also satisfies Equation (2).

Consider the sequence $\left\{\phi_{n}(x)\right\}$ defined as $n$ increases indefinitely and $h \rightarrow 0$. Assuming existence of a solution of Equation (3) it is easy to show that $\phi_{n}(x) \rightarrow y(x)$ uniformly in $\left(x_{0}, \zeta\right)$. For $y(x)$ is bounded and clearly also $\phi_{n}(x)$ is bounded in $\left(x_{0}, \zeta\right)$. For small enough $h$ we can make $\left|q(x)-q_{m}\right|<\epsilon$ in each sub-interval. By subtraction of Equation (3) from Equation (4) we have

$$
u^{\prime \prime}+q(x) u=M \omega(x),
$$

where $u=\phi_{n}(x)-y(x), M$ is a fixed positive number and $\omega(x)$ is a function such that $|\omega(x)|<\epsilon$ for every $x$ in $\left(x_{0}, \zeta\right)$. It follows from a standard theorem (de la Vallée Poussin op. cit., p. 142) that

$$
\left|\phi_{n}-y\right|+\left|\phi_{n}^{\prime}-y_{n}^{\prime}\right|<\epsilon\left\{e^{M\left(x-x_{0}\right)}-1\right\}
$$

i.e. $\phi_{n}(x)$ and $\phi_{n}^{\prime}(x)$ tend uniformly to $y(x)$ and $y^{\prime}(x)$ respectively. The modification involved if $q(x)$ is a bounded but not necessarily continuous function in $\left(x_{0}, \zeta\right)$ are trivial, but cases in which $q(x)$ is singular at some point but the solution still exists (e.g. a simple pole at $x=x_{0}$ with $y_{0}=0$ ) would require further consideration. The only material alteration in the treatment of Equation (1) if $p, q$ and $r$ are bounded in $\left(x_{0}, \zeta\right)$ is that $\phi_{n}(x)$ must now satisfy an equation

$$
\phi_{n}^{\prime \prime}(x)+p_{m} \phi_{n}^{\prime}(x)+q_{m} \phi_{n}(x)=r_{m}
$$

in each sub-interval $\left(x_{m-1}, x_{m}\right)$; and it is easy to see that the process may be extended to any linear differential equation and possibly to others as well.

These considerations establish that it is valid to found a step-by-step process on $\phi_{n}(x)$. By its definition, this function satisfies a linear differential equation with constant coefficients in a given interval and is therefore the same type of approximating function as used by Allen and Southwell. To some extent the constant coefficients are disposable. Allen and Southwell were concerned with boundary-value problems and made an a priori choice of these constants suited to their numerical process. We shall choose them in such a way that it is possible to develop the theory sufficiently to calculate explicitly the error terms involved in the approximations. It is sufficient to consider a typical interval, say $(0, h)$. We shall denote by $p_{0}, q_{0}$ and $r_{0}$ the numbers which represent $p$, $q$ and $r$ in this interval; these are subsequently to be defined.

2. Numerical integration formulae. Step-by-step formulae follow from the definition of $\phi_{n}(x)$. First consider Eq. (3) and put $\alpha_{0}=q_{0}^{1 / 2}$ in $(0, h)$. In this interval (cf. Eq. (6)), $\phi_{n}(x)$ satisfies

$$
\phi_{n}^{\prime \prime}(x)+\alpha_{0}^{2} \phi_{n}(x)=0,
$$


i.e.

$$
\phi_{n}(x)=\phi_{n}(0) \cos \alpha_{0} x+\frac{\phi_{n}^{\prime}(0)}{\alpha_{0}} \sin \alpha_{0} x .
$$

We consider $\phi_{n}(0)$ and $\phi_{n}^{\prime}(0)$ determined by continuity with values at the end of the previous interval. Putting $x=h$ gives

$$
\left.\begin{array}{l}
\phi_{n}(h)=\phi_{n}(0) \cos \alpha_{0} h+\frac{\phi_{n}^{\prime}(0)}{\alpha_{0}} \sin \alpha_{0} h \\
\phi_{n}^{\prime}(h)=\phi_{n}^{\prime}(0) \cos \alpha_{0} h-\alpha_{0} \phi_{n}(0) \sin \alpha_{0} h
\end{array}\right\}
$$

i.e. a pair of step-by-step formulae for calculating numerical approximations to $y(x)$ and $y^{\prime}(x)$. Since $x$ is assumed real $q_{0}$ is always real; if it is negative, then Eqs. (9) involve hyperbolic functions of real argument. It remains to assign a value to $q_{0}$. The one that we shall use is the integrated mean value of $q(x)$

$$
q_{0}=\frac{1}{h} \int_{0}^{h} q(x) d x .
$$

Next consider accuracy. If, for definiteness, we assume $q(x)$ continuous in $(0, h)$ then in general in this interval

$$
q(x)=q_{0}+O(h),
$$

no matter how we calculate $q_{0}$ and we can deduce a result similar to Eq. (7), that

$$
\left|\phi_{n}-y\right|+\left|\phi_{n}^{\prime}-y^{\prime}\right|=O(h) \text {, }
$$

i.e.

$$
\left|\phi_{n}^{\prime}-y^{\prime}\right|=O\left(h^{2}\right), \quad\left|\phi_{n}-y\right|=O\left(h^{3}\right) .
$$

On the other hand, consider the results of the following numerical example. Let $y$ satisfy

$$
y^{\prime \prime}+\left(3-x^{2}\right) y=0, \quad y(0)=0, \quad y^{\prime}(0)=1 \text {. }
$$

Using the formulae (9) with $h=\frac{1}{4}$ and calculating each $q_{0}$ from Eq. (10) we get, integrating to $x=1.5$, the results in Table 1 , where they are compared with values calcu-

TABLE 1

\begin{tabular}{l|l|l|l|l}
\hline$x$ & $\phi_{n}(x)$ & $\phi_{n}^{\prime}(x)$ & \multicolumn{1}{|c|}{$y(x)$} & \multicolumn{1}{c}{$y^{\prime}(x)$} \\
\hline 0 & 0 & 1 & 0 & 1 \\
0.25 & 0.242 & 0.908 & 0.242 & 0.909 \\
0.50 & 0.441 & 0.661 & 0.441 & 0.662 \\
0.75 & 0.567 & 0.328 & 0.566 & 0.330 \\
1.00 & 0.608 & -0.003 & 0.607 & 0.000 \\
1.25 & 0.574 & -0.261 & 0.572 & -0.258 \\
1.50 & 0.490 & -0.409 & 0.487 & -0.406 \\
\hline
\end{tabular}

lated from the known exact solution. The interesting feature of this table is that $\phi_{n}(x)$ and $\phi_{n}^{\prime}(x)$ are clearly correct to the same order of accuracy. Moreover, this is not an accidental result for it is a feature of the formulae (9) that they predict $\phi_{n}(h)$ and $\phi_{n}^{\prime}(h)$ 
both correct to order $h^{3}$, i.e. although the first of Eqs. (11) may be true in general in $(0, h)$, the case $x=h$ leads to a special result. We shall calculate the error term in the next section, but before doing so we state the formulae corresponding to Eq. (1). They are necessarily more complicated. In the typical intervial $(0, h), \phi_{n}(x)$ satisfies

$$
\phi_{n}^{\prime \prime}(x)+p_{0} \phi_{n}^{\prime}(x)+q_{0} \phi_{n}(x)=r_{0} .
$$

For convenience, introduce the function

$$
\chi_{n}(x)=\phi_{n}(x)-\frac{r_{0}}{q_{0}}
$$

and write

$$
\beta_{n}(0)=\chi_{n}^{\prime}(0)+\frac{1}{2} p_{0} \chi_{n}(0) .
$$

The formulae may then be written

$$
\left.\begin{array}{l}
\chi_{n}(h)=e^{-p_{0} h / 2}\left\{\chi_{n}(0) \cos \gamma_{0} h+\frac{\beta_{n}(0)}{\gamma_{0}} \sin \gamma_{0} h\right\} \\
\chi_{n}^{\prime}(h)=e^{-p_{0} h / 2}\left\{\beta_{n}(0) \cos \gamma_{0} h-\gamma_{0} \chi_{n}(0) \sin \gamma_{0} h\right\}-\frac{1}{2} p_{0} \chi_{n}(h)
\end{array}\right\},
$$

where

$$
\gamma_{0}=\left(q_{0}-\frac{1}{4} p_{0}^{2}\right)^{1 / 2} .
$$

Here $\chi_{n}(x)$ is a discontinuous function as we pass from interval to interval, since it is $\phi_{n}(x)$ that must preserve continuity.

As a practical illustration when a first derivative is present, consider an equation which occurs in Cochran's solution (5) for the viscous fluid motion near a rotating disc. The problem is

$$
G^{\prime \prime}-H G^{\prime}+H^{\prime} G=0, \quad G(0)=1, \quad G(\infty)=0,
$$

where $G(x)$ is the function to be found and $H(x)$ is a numerical function found from a second differential equation (itself depending on $G$, in fact). Cochran has tabulated $H(x)$ by steps of $x=0.1$ as far as $x=4.0$ and also gives the initial condition $G^{\prime}(0)=$ -0.616 corresponding to which he finds $G(\infty)=0$ to the required accuracy. If we take Cochran's $H$-values and initial $G^{\prime}$-value we can test Eqs. (13), with $\chi_{n}(x) \equiv \phi_{n}(x)$ in this case, by obtaining approximate values of $G(x)$ and $G^{\prime}(x)$. In the typical interval $(0, h)$ we take

$$
p_{0}=-\frac{1}{h} \int_{0}^{h} H d x, \quad q_{0}=\frac{1}{h}[H(h)-H(0)],
$$

following Eq. (10). Using a step $h=0.2$ the appropriate results, as far as $x=1.0$, are given in Table 2 . Beyond $x=1.0$ the comparison is very similar, and there is good theoretical reason for the approximation maintaining its accuracy as $x$ increases, for it is known that as $x \rightarrow \infty, H \rightarrow-c$, where $c>0$. The limiting form of the governing equation is

$$
G^{\prime \prime}+c G^{\prime}=0,
$$

a constant coefficient equation, so $\phi_{n}(x)$ becomes exact. As for our previous example 
TABLE 2

\begin{tabular}{|c|c|c|c|c|}
\hline \multirow[b]{2}{*}{$x$} & \multicolumn{2}{|c|}{ Eqs. (13) } & \multicolumn{2}{|c|}{ Cochran } \\
\hline & $\phi_{n}(x)$ & $\phi_{n}^{\prime}(x)$ & $G(x)$ & $G^{\prime}(x)$ \\
\hline 0 & 1.000 & -0.616 & 1.000 & -0.616 \\
\hline 0.2 & 0.879 & -0.598 & 0.878 & -0.599 \\
\hline 0.4 & 0.763 & -0.557 & 0.762 & -0.558 \\
\hline 0.6 & 0.657 & -0.504 & 0.656 & -0.505 \\
\hline 0.8 & 0.562 & -0.447 & 0.561 & -0.448 \\
\hline 1.0 & 0.479 & -0.390 & $0.468^{*}$ & -0.391 \\
\hline
\end{tabular}

${ }^{*}$ This value is an obvious misprint and should probably read 0.478 .

(and for identical reasons) the calculated values of $\phi_{n}(x)$ and $\phi_{n}^{\prime}(x)$ are both correct to the same order of accuracy.

3. Investigation of the error term. Let the solution $y(x)$ of Eq. (3) be defined in the typical interval $(0, h)$ with given initial values $y(0), y^{\prime}(0)$. Expand $q(x)$ in $(0, h)$ as the Fourier cosine series

$$
q(x)=a_{0}+2 \sum_{n=1}^{\infty} a_{n} \cos (n \pi x / h)
$$

and we may write Eq. (3) as

$$
y^{\prime \prime}+\left[a_{0}+2 \sum_{n=1}^{\infty} a_{n} \cos (n \pi x / h)\right] y=0 .
$$

With minor changes, this is equivalent to Hill's differential equation (cf. Whittaker and Watson (6)) and accordingly we assume a solution

$$
y(x)=e^{\mu x} \sum_{r=-\infty}^{\infty} c_{r} e^{i r \pi x / h}
$$

Substituting in Eq. (16) and equating coefficients of $\exp (\mu+i r \pi x / h)$ to zero we obtain the infinite set of linear algebraic equations

$$
\left(\mu+\frac{i n \pi}{h}\right)^{2} c_{n}+\sum_{r=-\infty}^{\infty} a_{n-r} c_{r}=0, \quad(n=\cdots,-2,-1,0,1,2, \cdots),
$$

where

Putting

$$
a_{-r}=a_{r} \quad(r=0,1,2, \cdots) .
$$

$$
\nu=\frac{\mu h}{\pi}, \quad b_{r}=\frac{h^{2}}{\pi^{2}} a_{r}
$$

these equations become

$$
\left[(\nu+i n)^{2}+b_{0}\right] c_{n}+\sum_{r=-\infty}^{\infty} b_{n-r} c_{r},(r \neq n),=0 .
$$

Eqs. (19) are homogeneous in $c_{r}$ and the values of $\nu$ for which solutions exist in which not all the $o_{r}$ are zero are given by the vanishing of a determinant $\Delta(i v)$ of Hill's type. 
Its properties are well known and need not further be discussed, since the only result of importance for present purposes is that if $\nu=\nu_{1}$ is a root, so is $\nu=-\nu_{1}$ and that these two are the only roots which lead to distinct solutions in the form of Eq. (17). We shall denote these roots simply by $\pm \nu$, to which correspond $\pm \mu$ by the first of Eqs. (18).

In obtaining solutions of Eqs. (19) we can without loss put $c_{0}=1$. Putting $n=0$ gives the equation

$$
\nu^{2}+b_{0}=\delta_{0}
$$

where

$$
\delta_{0}=-b_{1}\left(c_{1}+c_{-1}\right)-b_{2}\left(c_{2}+c_{-2}\right)-\cdots .
$$

and the remaining equations are

$$
\left(\delta_{0}-n^{2}+2 i v n\right) c_{n}+b_{n}=\delta_{n},
$$

where in general

$$
\delta_{n}=-\sum_{r=-\infty}^{\infty} b_{n-r} c_{r}, \quad(r \neq n, r \neq 0) .
$$

Consider now the orders (with respect to $h$ ) of the quantities involved. By definition

$$
a_{n}=\frac{1}{h} \int_{0}^{h} q(x) \cos (n \pi x / h) d x, \quad(n=0,1,2, \cdots),
$$

so $a_{0}=O(1)$ certainly, and hence $b_{0}=O\left(h^{2}\right)$. It is more difficult to be precise about the order of $a_{n}$ when $n \neq 0$ for the most general $q(x)$ we might consider but suppose, to be definite, that $q(x)$ is continuous in $(0, h)$. Then certainly $a_{n}=O(h)$ if $n \neq 0$, so $b_{n}=O\left(h^{3}\right)$.

It now follows that the first-order solution obtained by neglecting $\delta_{n}$ in Eq. (22), viz.

$$
c_{n} \sim \frac{b_{n}}{n^{2}+4 \nu^{2}}\left(1+\frac{2 i \nu}{n}\right)
$$

satisfies these equations formally with an error $O\left(h^{6}\right)$. For this approximation yields $c_{n}=O\left(h^{3}\right)$; moreover, $\sum\left|c_{n}\right|$ converges and the $b_{n}$ are bounded, so the $\delta_{n}$ exist. Hence $\delta_{n}=O\left(h^{6}\right)$. Furthermore, an even lower-order approximation to the solution of Eqs. (19) is obtained by neglecting even the approximate $c_{n}$ compared with $c_{0}$. Eq. (20) then gives

$$
\nu= \pm i b_{0}^{1 / 2}
$$

i.e.

$$
\mu= \pm i \alpha_{0}
$$

where

$$
\alpha_{0}=a_{0}^{1 / 2} .
$$

Putting $c_{0}=1, c_{r}=0,(r \neq 0)$, in Eq. (17) gives two approximate solutions corresponding to the roots (26) and we can combine them in the form

$$
\phi_{n}(x)=\phi_{n}(0) \cos \alpha_{0} x+\frac{\phi_{n}^{\prime}(0)}{\alpha_{0}} \sin \alpha_{0} x
$$


valid in $(0, h)$. Since $a_{0}$ is precisely the $q_{0}$ defined by Eq. (10), this is the $\phi_{n}(x)$ previously discussed.

To find the precise effect of the error terms on the step-by-step formulae (9), in general let the numbers $c_{n},(n= \pm 1, \pm 2, \cdots)$, be any set of coefficients satisfying Eqs. (19) with a corresponding root $\mu=i \alpha$ and write

$$
A_{n}=c_{n}+c_{-n} ; \quad B_{n}=c_{n}-c_{-n} .
$$

Then there is a corresponding fundamental solution $y=u(x)$ in the form of Eq. (17), where we can write

$$
u(x)=e^{i \alpha x}\left[f_{1}(x)+i f_{2}(x)\right]
$$

with

$$
\begin{aligned}
& f_{1}(x)=1+\sum_{n=1}^{\infty} A_{n} \cos (n \pi x / h), \\
& f_{2}(x)=\sum_{n=1}^{\infty} B_{n} \sin (n \pi x / h) .
\end{aligned}
$$

It is easy to show quite generally (cf. the approximation (25) to $c_{n}$ ) that the coefficients which satisfy Eqs. (19) with the root $\mu=-i \alpha$ involve only a change in sign in $B_{n}$, so a second solution is $y=v(x)$, where

$$
v(x)=e^{-i \alpha x}\left[f_{1}(x)-i f_{2}(x)\right] .
$$

The general solution can now be written in the form

$$
y(x)=C_{1}\left[f_{1}(x) \cos \alpha x-f_{2}(x) \sin \alpha x\right]+C_{2}\left[f_{1}(x) \sin \alpha x+f_{2}(x) \cos \alpha x\right] .
$$

Making it satisfy the initial conditions, we obtain

$$
C_{1}=\frac{y(0)}{f_{1}(0)}, \quad C_{2}=\frac{y^{\prime}(0)}{\alpha f_{1}(0)+f_{2}^{\prime}(0)},
$$

and step-by-step formulae follow if we put $x=h$ in $y(x)$ and $y^{\prime}(x)$.

Confining attention to the special case of the approximation (25), let us write

$$
S_{1}=\sum_{n=1}^{\infty} A_{n}, \quad S_{2}=\sum_{n=1}^{\infty}(-1)^{n+1} A_{n} .
$$

Then $f_{1}(0)=1+S_{1}, f_{1}(h)=1-S_{2}$ and from Eq. (25) we readily deduce that

$$
\alpha f_{1}(0)+f_{2}^{\prime}(0)=\alpha\left(1-S_{1}\right), \quad f_{2}^{\prime}(h)+\alpha f_{1}(h)=\alpha\left(1+S_{2}\right),
$$

so that the step-by-step formulae are

$$
\begin{aligned}
y(h) & =\frac{1-S_{2}}{1+S_{1}} y(0) \cos \alpha h+\frac{1-S_{2}}{\alpha\left(1-S_{1}\right)} y^{\prime}(0) \sin \alpha h \\
y^{\prime}(h) & =\frac{1+S_{2}}{1-S_{1}} y^{\prime}(0) \cos \alpha h-\frac{\alpha\left(1+S_{2}\right)}{1+S_{1}} y(0) \sin \alpha h .
\end{aligned}
$$

These formulae give only approximations to $y(h)$ and $y^{\prime}(h)$, of course, but it is not convenient to introduce special notation. For these approximations, formal justification. of the analysis follows readily enough if we assume $q(x)$ bounded in $(0, h)$. For then 
$\left|a_{n}\right|<K / n$, where $K$ is a positive constant, by well-known results of Fourier series, so $f_{1}(x)$ and $f_{1}^{\prime}(x)$ and a fortiori $f_{2}(x), f_{2}^{\prime}(x)$ converge absolutely and uniformly in $(0, h)$. The series (15) converges uniformly to $q(x)$ in any interior interval of $(0, h)$ in which $q(x)$ is continuous so, by Abel's test, $f_{1}^{\prime \prime}(x)$ and $f_{2}^{\prime \prime}(x)$ converge uniformly in the same interval justifying, to the given order of approximation, the satisfaction of the differential equation (16) by the solution (17); and only slight modifications in the arguments are necessary to extend the scope of Eqs. (29) when, subject to suitable initial conditions for $y(0)$ and $y^{\prime}(0), q(x)$ is any function satisfying Dirichlet's conditions in $(0, h)$, e.g. $q(x)=x^{-1 / 2}$ with the initial condition $y(0)=0$.

If we neglect $S_{1}$ and $S_{2}$ it is legitimate, within the same order of accuracy, to make $\alpha=\alpha_{0}=q_{0}^{1 / 2}$. It is now clear that $\phi_{n}(h)$ and $\phi_{n}^{\prime}(h)$ are both correct to the same order, being $O\left(h^{3}\right)$ if $q(x)$ is continuous. This interesting result, that there is no loss of accuracy in the differentiated function, is true only for $x=h$, of course, the reason being that whereas the differentiated series $f_{2}^{\prime}(x)$ makes a contribution to the step-by-step formulae when we put $x=h$, the differentiated series $f_{1}^{\prime}(x)$ does not. The former series depends only on $c_{n}-c_{-n}$ which, because of its dependence on $\nu$, always contains one higher power of $h$ than $c_{n}+c_{-n}$.

Next consider the modifications if we introduce a function $r(x)$ on the right hand side of Eq. (3). Let $y(x)$ denote the solution of this new equation in $(0, h)$ with initial values $y(0)$ and $y^{\prime}(0)$. Put $y(x)=\phi(x)+g(x)$, where $\phi(x)$ denotes the general solution of Eq. (3), and we have only to consider finding any particular solution of the equation

Let

$$
g^{\prime \prime}+q(x) g=r(x) \text {. }
$$

and assume a solution

$$
r(x)=d_{0}+2 \sum_{n=1}^{\infty} d_{n} \cos (n \pi x / h)
$$

$$
g(x)=c_{0}+\sum_{n=1}^{\infty} c_{n} \cos (n \pi x / h),
$$

i.e. we seek the particular solution having $g^{\prime}(0)=g^{\prime}(h)=0$. The $c_{n}$ in Eq. (31) are not the same as those in Eq. (17), of course, but there is no risk of confusion; the factor 2 is omitted from outside the summation for convenience. We now substitute in Eq. (30), multiply by $\cos (n \pi x / h)$ for each positive integer $n$ (including zero), and integrate from $x=0$ to $x=h$. It is easy to deduce the result

$$
\int_{0}^{h} g(x) q(x) \cos (n \pi x / h) d x=\frac{1}{2} h \sum_{p=0}^{\infty}\left(a_{n+p}+a_{n-p}\right) c_{p}
$$

so if we connect $a_{n}$ with $b_{n}$ as before and put $e_{n}=h^{2} d_{n} / \pi^{2}$ we find that Eq. (30) is reduced to the equivalent algebraic equations

$$
\left(b_{0}+b_{2 n}-n^{2}\right) c_{n}+\sum_{p=0}^{\infty}\left(b_{n+p}+b_{n-p}\right) c_{p},(p \neq n),=2 e_{n},
$$

which holds for all positive integers $n$, including zero. Assume for simplicity that both $q(x)$ and $r(x)$ are continuous in $(0, h)$ so that $b_{n}$ and $e_{n}$ are $O\left(h^{3}\right)$ if $n \neq 0$. Then a first approximation to the solution of Eqs. (32) is

$$
c_{0}=\frac{d_{0}}{a_{0}}, \quad c_{n}=O\left(h^{3}\right), \quad(n \neq 0),
$$


so that $g(x)=d_{0} / a_{0}$, with neglect of terms in $h^{3}$. Adding this particular solution to our previous approximation (to the same order of accuracy) to the general solution of Eq. (3), we arrive at an approximating function $\phi_{n}(x)$ which satisfies in $(0, h)$ the equation

$$
\phi_{n}^{\prime \prime}(x)+\alpha_{0}^{2} \phi_{n}(x)=r_{0},
$$

where

$$
r_{0}=d_{0}=\frac{1}{h} \int_{0}^{h} r(x) d x .
$$

We are therefore led to the same step-by-step formulae (13) or, more correctly, to the simplified form that results from putting $p_{0}=0$, viz.

$$
\left.\begin{array}{l}
\chi_{n}(h)=\chi_{n}(0) \cos \alpha_{0} h+\frac{\chi_{n}^{\prime}(0)}{\alpha_{0}} \sin \alpha_{0} h, \\
\chi_{n}^{\prime}(h)=\chi_{n}^{\prime}(0) \cos \alpha_{0} h-\alpha_{0} \chi_{n}(0) \sin \alpha_{0} h,
\end{array}\right\}
$$

with

$$
\chi_{n}(x)=\phi_{n}(x)-\frac{r_{0}}{q_{0}}
$$

as before. Again there is no additional loss of accuracy in the derivatives predicted by Eqs. (33) for, although a loss of accuracy clearly results when we differentiate $g(x)$, this does not affect the step-by-step formulae, since by hypothesis $g^{\prime}(0)=g^{\prime}(h)=0$.

A second approximation to the solution of Eqs. (32) can be obtained, neglecting terms of order only $h^{6}$. Here we must retain the terms in $b_{0}$ since in general these are $O\left(h^{2}\right)$. The approximation is

$$
c_{0}=\frac{d_{0}}{a_{0}}, \quad c_{n}=\frac{2\left(c_{0} b_{n}-e_{n}\right)}{n^{2}-b_{0}}, \quad(n=1,2, \cdots) .
$$

It leads to $g$-values at the ends of the interval

$$
g(0)=\frac{d_{0}}{a_{0}}+S_{3}, \quad g(h)=\frac{d_{0}}{a_{0}}-S_{4},
$$

where

$$
S_{3}=\sum_{n=1}^{\infty} c_{n}, \quad S_{4}=\sum_{n=1}^{\infty}(-1)^{n+1} c_{n}
$$

and, to this order of approximation, $c_{n}$ is given by the second of Eqs. (34). We can justify the validity of the approximate solutions much as before, and we need not consider this further.

The corresponding analysis relating to the differential equation (1) is similar but a little more complicated. If $r(x)=0$ we make the same assumption (Eq. (17)) for $y(x)$ and arrive at the algebraic equations

$$
\left[(\nu+i n)^{2}+b_{0}^{\prime}(\nu+i n)+b_{0}\right] c_{n}+\sum_{r=-\infty}^{\infty}\left[b_{n-r}^{\prime}(\nu+i r)+b_{n-r}\right] c_{r},(r \neq n),=0
$$

which holds for $n=0, \pm 1, \pm 2, \cdots$ and in which $\nu$ and $b_{n}$ have their previous significance 
while $b_{n}^{\prime}=h a_{n}^{\prime} / \pi$, the $a_{n}^{\prime}$ being Fourier coefficients of the series (15) when $p(x)$ replaces $q(x)$. Again we may verify that a first approximation is

$$
c_{0}=1, \quad c_{n}=O\left(h^{3}\right), \quad(n \neq 0),
$$

provided that $p(x)$ and $q(x)$ are continuous in $(0, h)$. The approximation to the $c_{n}$ comes from the equations

$$
\left[(\nu+i n)^{2}+b_{0}^{\prime}(\nu+i n)+b_{0}\right] c_{n}+\nu b_{n}^{\prime}+b_{n}=0
$$

and although $b_{n}^{\prime}=O\left(h^{2}\right)$ in general, $\nu=O(h)$ so $c_{n}=O\left(h^{3}\right)$. Corresponding to the approximation (36) we determine two $\nu$-values from the equation

$$
\nu^{2}+b_{0}^{\prime} \nu+b_{0}=0
$$

i.e.

$$
\mu^{2}+a_{0}^{\prime} \mu+a_{0}=0 .
$$

Subsequent analysis follows more or less as before, although it is more complicated; we shall not give the details. When $r(x) \neq 0$ we get the same particular solution $g(x)=$ $d_{0} / a_{0}$, with an error of order $h^{3}$, to the complete equation (1). Adding it to the approximate general solution of the reduced equation, we arrive at the approximating function

$$
\phi_{n}(x)=C_{1} e^{\mu_{1} x}+C_{2} e^{\mu_{2} x}+\frac{r_{0}}{q_{0}},
$$

and hence to the step-by-step formulae (13). The details of the analysis show that these formulae again predict approximations to $\phi_{n}(h)$ and $\phi_{n}^{\prime}(h)$ which are both correct to terms of order $h^{3}$.

4. Higher order approximations. If we retain the error terms $S_{1}$ and $S_{2}$ in Eqs. (29), we will obtain a more accurate approximation to the solution of Eq. (3). We can express $S_{1}$ and $S_{2}$ more conveniently in terms of definite integrals by making use of the results, valid in $(0, h)$,

$$
\cosh 2 \mu x=t_{0}+2 \sum_{n=1}^{\infty} t_{n} \cos (n \pi x / h)
$$

and

$$
\cosh 2 \mu(x-h)=t_{0}+2 \sum_{n=1}^{\infty}(-1)^{n} t_{n} \cos (n \pi x / h),
$$

where

$$
t_{n}=\frac{2 \nu h \sinh 2 \nu h}{\pi^{2}} \frac{(-1)^{n}}{n^{2}+4 \nu^{2}}, \quad(n=0,1,2, \cdots) .
$$

The summations required are

$$
S_{1}=\frac{2 h^{2}}{\pi^{2}} \sum_{n=1}^{\infty} \frac{a_{n}}{n^{2}+4 \nu^{2}} \quad \text { and } S_{2}=\frac{2 h^{2}}{\pi^{2}} \sum_{n=1}^{\infty} \frac{(-1)^{n+1} a_{n}}{n^{2}+4 \nu^{2}}
$$

To find $S_{1}$, multiply the left hand side of Eq. (39) by $q(x)$ and the right hand side by the equivalent series (15) and integrate each side from $x=0$ to $x=h$. A similar operation 
applied to Eq. (38) gives $S_{2}$, and both operations are valid by well-known results of Fourier series. Simplifying and putting $\mu=i \alpha$ we obtain

$$
\left.\begin{array}{l}
S_{1}=\frac{a_{0}}{4 \alpha^{2}}-\frac{1}{2 \alpha \sin 2 \alpha h} \int_{0}^{h} q(x) \cos 2 \alpha(x-h) d x, \\
S_{2}=\frac{1}{2 \alpha \sin 2 \alpha h} \int_{0}^{h} q(x) \cos 2 \alpha x d x-\frac{a_{0}}{4 \alpha^{2}}
\end{array}\right\}
$$

It remains to consider the accuracy of the formulae (29) in a little more detail. The error involved in Eq. (25) is in general $O\left(h^{6}\right)$ but we have to examine whether any accuracy is lost when we differentiate the corresponding approximate solution for $y(x)$. We shall omit the details, merely quoting the result that, as in the case of our first approximation, the error term in $c_{n}-c_{-n}$ appears to one higher power in $h$ than in $c_{n}+c_{-n}$ and this is sufficient to ensure that there is no loss of accuracy in the differentiated solution at $x=h$. But we have yet to consider the effect of making the approximation (26) to $\mu$, i.e. we have to find an appropriate value of $\alpha$ to substitute in Eqs. (29) and (40). Put $\nu=i h \alpha / \pi$ in Eq. (20) and substitute in $\delta_{0}$ using Eq. (25). This gives

$$
\alpha^{2} \sim a_{0}+\frac{2 h^{2}}{\pi^{2}} \sum_{n=1}^{\infty} \frac{a_{n}^{2}}{n^{2}-4 h^{2} \alpha^{2} / \pi^{2}}
$$

i.e.

$$
\alpha^{2}=a_{0}+O\left(h^{4}\right) \text {. }
$$

Now let us expand the terms $\cos \alpha h$ and $\sin \alpha h$ in Eqs. (29) in powers of $h$, thus

$$
\left.\begin{array}{rl}
y(h) & =\frac{1-S_{2}}{1+S_{1}} y(0)\left(1-\frac{1}{2} \alpha^{2} h^{2}+\cdots\right)+\frac{1-S_{2}}{1-S_{1}} y^{\prime}(0)\left(h-\frac{1}{6} \alpha^{2} h^{3}+\cdots\right), \\
y^{\prime}(h) & =\frac{1+S_{2}}{1-S_{1}} y^{\prime}(0)\left(1-\frac{1}{2} \alpha^{2} h^{2}+\cdots\right)-\frac{1+S_{2}}{1+S_{1}} y(0)\left(\alpha^{2} h-\frac{1}{6} \alpha^{4} h^{3}+\cdots\right) .
\end{array}\right\}
$$

Assuming for the moment that we have computed accurate enough values of $S_{1}$ and $S_{2}$, it is clear that putting $\alpha=a_{0}^{1 / 2}$ in the first of Eqs. (42) will involve only an error of order $h^{6}$, but in the second the term $\alpha^{2} h$ will lead to an error of order $h^{5}$. If we want to avoid this we must compute a more accurate value of $\alpha^{2}$ using Eq. (41) and, moreover, this value will then serve to substitute in Eqs. (40) to find $S_{1}$ and $S_{2}$ without any further drop in accuracy below the general level $h^{6}$ we are working to. The series in Eq. (41) must be very rapidly convergent and the first term or two will suffice.

It is interesting to note that the higher order approximation to the solution $y(x)$ does not involve any further differentiations beyond the order of the differential equation; and although we have assumed $q(x)$ continuous for the sake of stating definite orders of accuracy this is not necessary. In most practical problems, however, $q(x)$ will be continuous in a given interval $(0, h)$. It may then be convenient to evaluate the integrals in Eqs. (40) by numerical quadrature, e.g. if we assume $q(x)$ to be parabolic in $(0, h)$ we can easily obtain the results

$$
\begin{aligned}
& S_{1}+S_{2} \sim \frac{1}{4 \alpha^{2}}\left(1-\frac{\tan \alpha h}{\alpha h}\right)[q(h)-q(0)] \\
& S_{1}-S_{2} \sim \frac{1}{6 \alpha^{2}}\left[\frac{3(1-\alpha h \cot \alpha h)}{\alpha^{2} h^{2}}-1\right]\left[q(0)+q(h)-2 q\left(\frac{1}{2} h\right)\right],
\end{aligned}
$$


or, if we expand $\tan \alpha h$ and cot $\alpha h$ in powers of $h$ and neglect terms in $h^{6}$ and above, these become

$$
\left.\begin{array}{l}
S_{1}+S_{2} \sim \frac{1}{12} h^{2}\left(1+\frac{2}{5} \alpha^{2} h^{2}\right)[q(0)-q(h)] \\
S_{1}-S_{2} \sim \frac{1}{90} h^{2}\left(1+\frac{1}{21} \alpha^{2} h^{2}\right)\left[q(0)+q(h)-2 q\left(\frac{1}{2} h\right)\right] .
\end{array}\right\}
$$

The results are expressed in this way for convenience but, in fact, since

$$
\left(1+S_{1}\right)^{-1}=1-S_{1}+O\left(h^{6}\right)
$$

and

$$
\left(1-S_{1}\right)^{-1}=1+S_{1}+O\left(h^{6}\right),
$$

we can express Eqs. (29) entirely in terms of $S_{1}+S_{2}, S_{1}-S_{2}$; and the advantage of Eqs. (43) is that we can calculate explicitly the first-order error terms very simply before we start a computation.

Similar results apply to the terms $S_{3}$ and $S_{4}$ in Eqs. (35) when the $c_{n}$ are given by the second of Eqs. (34). We have only to replace $2 \alpha$ by $b_{0}^{1 / 2}$ and $q(x)$ by the function $c_{0} q(x)-$ $r(x)$ (with $c_{0}$ given by the first of Eqs. (34)) in either of Eqs. (40) or (43) to get corresponding results involving $S_{3}$ and $S_{4}$.

We shall illustrate with two numerical examples. In the first we compute an improved solution to the problem (12) using the same step $h=0.25$. The results are shown in the

\begin{tabular}{|c|c|c|c|c|}
\hline \multirow[b]{2}{*}{$x$} & \multicolumn{2}{|c|}{ Eqs. (29) } & \multicolumn{2}{|c|}{ Exact } \\
\hline & $y(x)$ & $y^{\prime}(x)$ & $y(x)$ & $y^{\prime}(x)$ \\
\hline 0 & 0 & 1 & 0 & 1 \\
\hline 0.25 & 0.242308 & 0.908656 & 0.242308 & 0.908657 \\
\hline 0.50 & 0.441249 & 0.661872 & 0.441248 & 0.661873 \\
\hline 0.75 & 0.566129 & 0.330242 & 0.566130 & 0.330243 \\
\hline 1.00 & 0.606530 & -0.000001 & 0.606531 & 0.000000 \\
\hline 1.25 & 0.572291 & -0.257533 & 0.572292 & -0.257532 \\
\hline 1.50 & 0.486977 & -0.405817 & 0.486978 & -0.405815 \\
\hline
\end{tabular}
following table, where they are compared with values calculated from the exact solution.

TABLE 3

The value of $\alpha$ in each interval has been calculated from Eq. (41). It is easily shown that in the $m$-th interval from the start

$$
a_{n}=2 h^{2}\left[m-1+(-1)^{n+1} m\right] / n^{2} \pi^{2}, \quad(n=1,2, \cdots),
$$

so that computation from Eq. (41) is very simple, e.g. even in the final interval the computation is, to six decimals,

$$
\alpha^{2}=1.104167+0.000247+0.000003,
$$

the first term being the value of $a_{0}$. 
As an example of a non-homogeneous equation we have computed an approximate solution to the problem

$$
y^{\prime \prime}+\left(3-x^{2}\right) y=2, \quad y(0)=0, \quad y^{\prime}(0)=1 .
$$

Although here $r(x)$ is constant, this is hardly a special simplification as the operative function in finding $S_{3}$ and $S_{4}$ is $c_{0} q(x)-r(x)$, which is not constant. The solution has quite different properties from that of Eq. (12); as a comparison solution we have obtained a power series solution and we believe the values tabulated from it to be correct to five decimals. They are shown, along with values computed using Eqs. (29) and (35), in Table 4. Here the solution appears to be becoming a little unstable as $x$ increases.

TABLE 4

\begin{tabular}{|c|c|c|c|c|}
\hline \multirow[b]{2}{*}{$x$} & \multicolumn{2}{|c|}{ Eqs. (29) and (35) } & \multicolumn{2}{|c|}{ Power series } \\
\hline & $y(x)$ & $y^{\prime}(x)$ & $y(x)$ & $y^{\prime}(x)$ \\
\hline 0 & 0 & 1 & 0 & 1 \\
\hline 0.25 & 0.30385 & 1.39337 & 0.30385 & 1.39337 \\
\hline 0.50 & 0.67651 & 1.54736 & 0.67651 & 1.54735 \\
\hline 0.75 & 1.05920 & 1.48397 & 1.05920 & 1.48396 \\
\hline 1.00 & 1.40811 & 1.29747 & 1.40812 & 1.29745 \\
\hline 1.25 & 1.70913 & 1.12569 & 1.70908 & 1.12562 \\
\hline 1.50 & 1.98475 & 1.11986 & 1.98470 & 1.11969 \\
\hline
\end{tabular}

We have not attempted to obtain a higher order approximation to the more general equation (1). It is possible, but more complicated, and in any case we can always make a well-known transformation of the dependent variable to remove the first-derivative term. For example, Cochran's problem (Eq. (14)) can be transformed, by making

to the problem

$$
G=g \exp \left[\frac{1}{2} \int_{0}^{t} H d t\right]
$$

$$
g^{\prime \prime}+\left(\frac{3}{2} H^{\prime}-\frac{1}{4} H^{2}\right) g=0, \quad g(0)=1, \quad g^{\prime}(0)=-0.616,
$$

and using this form we have found that the results in Table 2 can be reproduced by means of Eqs. (29) with a step $h=0.8$. Even if it is inconvenient to make a permanent transformation of the dependent variable we can use the device of transforming locally and immediately returning to the original variable, which we have explained in our recent paper (2).

5. Summary. This paper is by no means very general in detail, as we have restricted our considerations to linear second-order differential equations. Certain extensions could at once be made, for example to the important fourth-order problem.

$$
\frac{d^{2}}{d x^{2}}\left[p(x) \frac{d^{2} y}{d x^{2}}\right]+q(x) y=0
$$

with suitable initial conditions, since this problem is expressible as a simultaneous pair of linear second-order equations. In theory the process can be applied to any linear 
differential equation, although the details would need consideration. Certain non-linear equations could also be treated. For example, the well-known Blasius' problem

$$
y^{\prime \prime \prime}+y y^{\prime \prime}=0, \quad y(0)=y^{\prime}(0)=0, \quad y^{\prime \prime}(0)=k
$$

could be treated by using a function $\phi_{n}(x)$ which satisfies, in a typical interval $(0, h)$, the equation

$$
\phi_{n}^{\prime \prime \prime}(x)+\rho_{0} \phi_{n}^{\prime \prime}(x)=0,
$$

setting up step-by-step formulae in the manner we have indicated in this paper, and determining $\rho_{0}$ for the given interval from the transcendental equation

$$
h \rho_{0}=\int_{0}^{h} \phi_{n}(x) d x .
$$

The object of the paper, therefore, is to suggest a possible approach which might sometimes be fruitful, since it must be agreed that no method of numerical integration can hope to solve every problem with uniformly satisfactory results.

\section{References}

1. D. N. de G. Allen and R. V. Southwell, Relaxation method applied to determine the motion, in two dimensions, of a viscous fluid past a fixed cylinder, Quart. J. Mech. 8, 129-145 (1955)

2. S. C. R. Dennis, Finite differences associated with second-order differential equations, Quart. J. Mech. 13, 487-507 (1960)

3. E. L. Ince, Ordinary differential equations, Dover, New York, 1926

4. C. de la Vallée Poussin, Cours d'analyse infinitesimale 2, Louvain, 1928, pp. 127-151

5. W. G. Cochran, The flow due to a rotating disc, Proc. Camb. Phil. Soc. 30, 365-375 (1934)

6. E. T. Whittaker and G. N. Watson, Modern analysis, The University Press, Cambridge, 1935, pp. 413-417 